Document downloaded from:

http://hdl.handle.net/10251/125100

This paper must be cited as:

Si, G.; Liao, H.; Yu, D.; Llopis Albert, C. (2018). Interval-valued 2-tuple hesitant fuzzy linguistic term set and its application in multiple attribute decision making. Journal of Intelligent \& Fuzzy Systems. 34(6):4225-4236. https://doi.org/10.3233/JIFS-171967

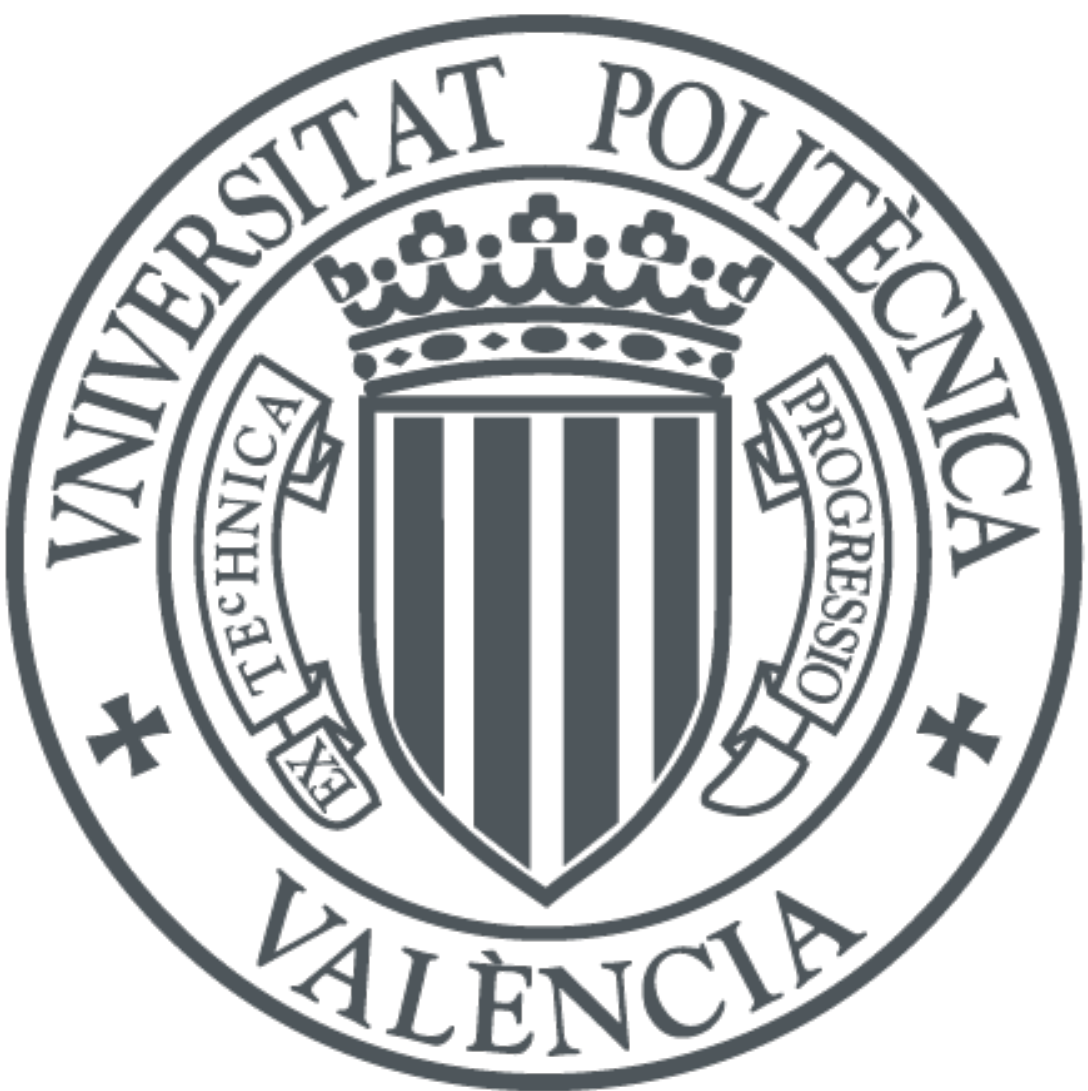

The final publication is available at

http://doi.org/10.3233/JIFS-171967

Copyright IOS Press

Additional Information 


\title{
Interval-valued 2-tuple hesitant fuzzy linguistic term set and its application in multiple attribute decision making
}

\author{
Guangsen $\mathrm{Si}^{1}$, Huchang Liao, ${ }^{1, *}$, Dejian $\mathrm{Yu}^{2}$, Carlos Llopis-Albert ${ }^{3}$ \\ ${ }^{1}$ Business School, Sichuan University, Chengdu 610064, China \\ ${ }^{2}$ School of Information, Zhejiang University of Finance and Economics, Hangzhou, Zhejiang 310018, China \\ ${ }^{3}$ Universitat Politècnica de València, C/ Camí de Vera s/n, 46022 Valencia, Spain
}

\begin{abstract}
The hesitant fuzzy linguistic term sets can retain the completeness of linguistic information elicitation by assigning a set of possible linguistic terms to a qualitative variable. However, sometimes experts cannot make sure that the objects attain these possible linguistic terms but only provide the degrees of confidence to express their hesitant cognition. Given that the interval numbers can denote the possible membership degrees that an object belongs to a set, it is suitable and convenient to provide an interval-valued index to measure the degree of a linguistic variable to a given hesitant fuzzy linguistic term set. Inspired by this idea, we introduce the concept of interval-valued 2-tuple hesitant fuzzy linguistic term set (IV2THFLTS) based on the interval number and the hesitant fuzzy linguistic term set. Then, we define some interval-valued 2-tuple hesitant fuzzy linguistic aggregation operators. Afterwards, to overcome the instability of subjective weights, we propose a method to compute the weights of attributes. For the convenience of application, a method is given to solve the multiple attribute decision making problems with IV2THFLTSs. Finally, a case study is carried out to validate the proposed method, and some comparisons with other methods are given to show the advantages of the proposed method.
\end{abstract}

Keywords: Hesitant fuzzy linguistic term sets, interval numbers, interval-valued 2-tuple hesitant fuzzy linguistic term set, aggregation operators, weight determining method, oversea investment evaluation

\section{Introduction}

Torra [1] introduced the hesitant fuzzy set (HFS) to express the membership degrees that an element belongs to a set as some discrete values in $[0,1]$. The HFS is useful in representing the hesitancy of decision-makers (DMs)' cognition when determining the evaluation values $[2,3]$. It has attracted many researchers' attention $[4,5,6]$. However, the HFS can only be used to represent the quantitative information. To retain the completeness of linguistic elicitation based on the fuzzy linguistic approach [7], motivated by the HFS, Rodríguez et al. [8] introduced the hesitant fuzzy linguistic term set (HFLTS) as an ordered finite subset of a consecutive linguistic term set (LTS). Liao et al. [9] redefined the HFLTS in

*Corresponding author. E-mail address: liaohuchang@163.com (H.C. Liao), guangsensi@163.com (G.S.Si). mathematical representation and called its elements as hesitant fuzzy linguistic elements (HFLEs). Since the HFLTS can retain the completeness of linguistic information elicitation, it has been a hot research topic [10]. Wei et al. [11] defined the operations on HFLEs based on the convex combination and compared the HFLEs based on the possibility degree formulas. To enhance the applicability of HFLTSs, different types of distance and similarity measures between HFLTSs were investigated $[12,13]$. Liao et al. [14] developed a hesitant linguistic VIKOR method to solve the multiple attribute decision making (MADM) problems within the context of HFLTSs and the criteria conflict with each other. Zhang et al. [15] applied the hesitant linguistic VIKOR method to the inpatient admission assessment process in West China Hospital. Rodríguez et al. [16] proposed a new linguistic group decision model to promote the elicitation of flexible 
and rich linguistic information based on the HFLTS. Liao et al. [17] developed two methods for hesitant linguistic MADM problems based on the ELECTRE II method.

Although the HFLTS is useful in representing the complex linguistic expressions, it is limited in some cases to represent comprehensive linguistic information $[18,19]$. Thus, many scholars extended the HFLTS into different variations. Wang [20] generalized the HFLTS by enabling any non-consecutive linguistic terms in them, and referred it as the extended HFLTS (EHFLTS). Zhang and $\mathrm{Wu}$ [21] proposed the concept of the hesitant fuzzy linguistic set (HFLS) by combining the HFS and the fuzzy linguistic approach. Chen et al. [22] proposed the proportional HFLTS, which includes the proportional information of each generalized linguistic term. Lin et al. [23] proposed the concepts of HFLS and hesitant fuzzy uncertain linguistic set (HFULS), but the concept of HFLS they introduced is different from that defined by Zhang and $\mathrm{Wu}$ [21]. Wei [24] proposed the concept of interval valued HFULS based on the HFS and the uncertain LTS. Wang et al. [25] proposed the concept of interval-valued HFLS (IVHFLS) based on the interval-valued HFS. Due to the hesitancy and uncertainty of DMs' cognition, Meng et al. [26] introduced the linguistic interval HFS (LIHFS) based on the linguistic hesitant fuzzy set (LHFS) [27], where the membership degrees of linguistic terms are intervals rather than real numbers. The LHFS not only gives the possible linguistic terms of a linguistic variable but also considers the possible membership degree of each linguistic term. To extend the applicability of LHFSs, Zhu et al. [28] proposed the concept of the comprehensive cloud of LHFSs. As shown in Table 1, a wide range of concepts were proposed in the literature.

Considering the powerfulness of HFLTS, it is flexible for DMs to provide their opinions by HFLTS but sometimes they cannot make sure that the objects attain these possible linguistic terms but only provide the degrees of confidence to express their hesitant cognition. Due to the complexity of MADM problems and the subjective uncertainty of DMs, it is difficult for DMs to express membership degree with the precise values. $\mathrm{Xu}$ and $\mathrm{Da}$ [29] first introduced the concept of interval number and defined the operations of interval numbers. As Chen et al. [30] noted, the precise membership degrees in the form of some discrete values in $[0,1]$ are sometimes hard to be obtained. It may be flexible for DMs to express the membership degrees with an interval number within $[0,1]$. Given that the interval numbers can denote the possible membership degrees that an element belongs to a given set, it is suitable and convenient to provide an interval-valued index to measure the degree or intensity of a linguistic variable to a given HFLTS. Inspired by this idea, in this paper, we extend the HFLTS to the interval-valued 2-tuple hesitant fuzzy linguistic term set (IV2THFLTS) based on the interval number and the HFLTS. Then, we define several IV2THFL aggregation operators for solving MADM problems. To overcome the instability of subjective weights, a method is proposed to compute the weights of attributes. For the convenience of application, a method is given to solve the MADM problems with IV2THFLTSs.

Table 1. A comparison of different extended concepts

\begin{tabular}{lllll}
\hline Concepts & Year & Reference & Representation form & Example \\
\hline HFLTS & 2012 & {$[8]$} & $\left\{<x_{i}, h_{S}\left(x_{i}\right)>\mid x_{i} \in X\right\}$ & $\left\{s_{3}, s_{4}, s_{5}\right\}$ \\
PHFLTS & 2016 & {$[22]$} & $\left\{\left(s_{i}, p_{i}\right) \mid s_{i} \in S, i=0,1, \ldots, g\right\}$ & $\left\{\left(s_{3}, 0.3\right),\left(s_{5}, 0.5\right)\right\}$ \\
HFLS & 2014 & {$[23]$} & $\left(<x, s_{\theta(x)}, h_{A}(x)>\mid x \in X\right)$ & $<s_{2},\{0.3,0.5\}>$ \\
HFULS & 2014 & {$[23]$} & $\left(<x, \tilde{s}_{\theta(x)}, h_{\tilde{A}}(x)>\mid x \in X\right)$ & $<\left[s_{2}, s_{3}\right],\{0.3,0.5\}>$ \\
IVHFLS & 2014 & {$[25]$} & $\left\{<x, s_{\theta(x)}, \Gamma_{A}(x)>\mid x \in X\right\}$ & $<s_{2},[0.3,0.5],[0.6,0.7]>$ \\
IVHFULS & 2016 & {$[24]$} & $\left(<x, \tilde{s}_{\theta(x)}, h_{\tilde{A}}(x)>\mid x \in X\right)$ & $<\left[s_{2}, s_{4}\right],\{0.3,0.5,0.8\}>$ \\
LHFS & 2014 & {$[27]$} & $\left\{\left(s_{\theta(i)}, l h\left(s_{\theta(i)}\right) \mid s_{\theta(i)} \in S\right)\right\}$ & $\left(s_{1}, 0.2,0.3\right)$ \\
LIHFS & 2016 & {$[26]$} & $\left\{\left(s_{\theta(i)}, \bar{h}\left(s_{\theta(i)}\right) \mid s_{\theta(i)} \in S\right)\right\}$ & $\left(s_{3},[0.2,0.3],[0.4,0.5]\right)$
\end{tabular}

The main contributions of this paper are summarized as follows:

(1) We extend the HFLTS to the IV2THFLTS which expresses the evaluation information more flexibly by depicting the interval-valued membership degrees in the form of interval numbers. It can retain the completeness of linguistic information given by the DMs and thus effectively enhance the accuracy of 
decision results.

(2) We define some generalized aggregation operators for IV2THFLEs. With these operators, DMs can choose different values of parameter $\lambda$ to express their preference.

(3) We propose a method to obtain the weights of attributes to overcome the instability of subjective weights based on the differences of these attributes. The weights obtained in this paper can improve the accuracy of decision results.

(4) We propose a method to solve the MADM problems with the IV2THFLTSs. We illustrate the procedure by a case study concerning the oversea investment evaluation.

The remainder of this paper is organized as follows. In Section 2, some basic knowledge of interval number, HFLTS and HFULS are reviewed. In Section 3, we define the concept of IV2THFLTSs and their operations. In Section 4, some aggregation operators for IV2THFLEs are defined. We propose a method to obtain the weights of attributes and then develop a method to solve the MADM problems under IV2THFL environment. Section 5 illustrates the applicability of the proposed method. Some conclusions are drawn in Section 6.

\section{Preliminaries}

\subsection{Interval numbers}

It may be difficult for DMs to give the precise membership degree of an element to a set. However, it is easy to give the interval-valued membership degree $\tilde{a}=\left[a^{L}, a^{U}\right]$. Especially, $\tilde{a}$ is a real number if $a^{L}=a^{U}$. Let $\tilde{a}=\left[a^{L}, a^{U}\right]$ and $\tilde{b}=\left[b^{L}, b^{U}\right]$ be two interval numbers and $\lambda \geq 0$. Then,

(1) $\tilde{a}+\tilde{b}=\left[a^{L}+b^{L}, a^{U}+b^{U}\right]$;

(2) $\lambda \tilde{a}=\left[\lambda a^{L}, \lambda a^{U}\right]$;

(3) $\tilde{a} \cdot \tilde{b}=\left[a^{L} \cdot b^{L}, a^{U} \cdot b^{U}\right]$;

(4) $\tilde{a}^{\lambda}=\left[\left(a^{L}\right)^{\lambda},\left(a^{U}\right)^{\lambda}\right]$.

The possibility degree of $\tilde{a} \geq \tilde{b}$ is defined as [29]:

$$
p(\tilde{a} \geq \tilde{b})=\max \left\{1-\max \left(\frac{b^{U}-a^{L}}{a^{U}-a^{L}+b^{U}-b^{L}}, 0\right), 0\right\}
$$

\subsection{HFLTS}

Let $S=\left\{s_{t} \mid t=-\tau, \ldots, 0, \ldots, \tau\right\} \quad$ be a finite LTS, satisfying: $s_{i}>s_{j}$, if $i>j$. To retain the completeness of information, $S$ is extended to $\bar{S}=\left\{s_{t} \mid t \in[-\tau, \tau]\right\} \quad$ [29]. To improve the accuracy of linguistic information representation, Rodríguez et al. [8] proposed the HFLTS, which is an ordered finite subset of consecutive linguistic terms of $S$. Later, Liao et al. [9] redefined mathematically as

$$
H_{S}=\left\{<x, h_{S}(x)>\mid x \in X\right\}
$$

where $\quad h_{S}(x)=\left\{s_{\phi_{l}}(x) \mid \quad s_{\phi_{l}}(x) \in S, l=1, \ldots, L\right\}$ denotes the possible membership degrees of $x$ to $S$. For convenience, $h_{S}(x)$ is called the HFLE.

The upper bound $H_{S^{+}}=\max \left\{s_{i}, s_{j}\right\}$ and the lower bound $H_{S^{-}}=\min \left\{s_{i}, s_{j}\right\}$ of $H_{S}$ are introduced to define the envelope of $H_{S}$ [8]. The envelope of a HFLTS, $e n v\left(H_{S}\right)$, is a linguistic interval, where

$$
\operatorname{env}\left(H_{S}\right)=\left[H_{S^{-}}, H_{S^{+}}\right], H_{S^{-}} \leq H_{S^{+}}
$$

\subsection{HFULS}

$\mathrm{Xu}$ [31] proposed the concept of uncertain linguistic variable as interval linguistic terms. Inspired by the idea of HFS which represents the membership degree of an element to a set in multiple values, Lin et al. [23] proposed the HFULS by combining the uncertain linguistic variable and HFS. Let $\tilde{S}$ be a set of uncertain linguistic terms. A HFULS on $X$ is in form of:

$$
\tilde{A}=\left(x,<\tilde{S}_{\theta(x)}, h_{\tilde{A}}(x)>\mid x \in X\right)
$$

where $h_{\tilde{A}}(x)$ is a set of some values in $[0,1]$, denoting the possible membership degrees that element $x$ belongs to an uncertain linguistic term $\tilde{S}_{\theta(x)}$. We call $<\tilde{s}_{\theta(x)}, h_{\tilde{A}}(x)>=<\left[s_{\theta^{L}(x)}, s_{\theta^{R}(x)}\right]$, $h_{\tilde{A}}(x)>$ the hesitant fuzzy uncertain linguistic element (HFULE).

\section{The IV2THFLTSs and their operations}

By combining interval number with HFLTE, we can introduce the concept of IV2THFLTS. Then we shall define the operations and comparison laws of IV2THFLEs in this section. 


\subsection{IV2THFLTS}

Considering that DMs cannot make sure that an object belongs to a HFLE, we introduce an interval-valued index to measure the degree of a linguistic variable to a given HFLE. In this sense, it is natural to define the IV2THFLTS.

Definition 1. Let $X=\left\{x_{1}, x_{2}, \ldots, x_{n}\right\}$ be a reference set and $h_{S}(x)$ be a HFLTS on $S$. The IV2THFLTS $A$ in $X$ is defined as:

$$
A=\left\{<x, h_{S}(x), \tilde{I}_{A}(x)>\mid x \in X\right\}
$$

where $\tilde{I}_{A}(x)$ is a closed subinterval of $[0,1]$, denoting the possible interval-valued membership degree of $x$ to $h_{S}(x)$. For convenience, $e=<h_{S}, \tilde{I}_{A}>$ is called an interval-valued 2-tuple hesitant fuzzy linguistic element (IV2THFLE). $A$ is the collection of all IV2THFLEs. When $\tilde{I}_{A}=[1,1]$, the IV2THFLTS is reduced to the HFLTS.

The HFULS is composed of the HFS and the uncertain linguistic variable, where the membership degree of linguistic variable $x \in X$ to the uncertain linguistic set $\tilde{S}_{\theta(x)}$ is represented by the HFS. Compared with the HFULS, the proposed IV2THFLTS consists of HFLTS and interval number. Experts are often unable to determine some precise membership degrees in the form of HFS. However, the interval number can accurately denote the membership degrees of linguistic variables to the HFLTS, which is convenient for DMs to provide the membership degrees. In addition, the HFLTS can avoid linguistic information loss in decision process.

\subsection{Operations of IV2THFLEs}

Motivated by the operations of HFULSs [21] and interval numbers [29], we develop some operations of IV2THFLEs.

Definition 2. Let $e=<h_{S},\left[r^{L}, r^{U}\right]>\quad, \quad e_{1}=$ $<h_{S}^{1},\left[r_{1}^{L}, r_{1}^{U}\right]>$ and $e_{2}=<h_{S}^{2},\left[r_{2}^{L}, r_{2}^{U}\right]>$ be three IV2THFLEs and $\lambda \geq 0$. Then, we have

(1) $e_{1} \oplus e_{2}=<U_{s_{\alpha} \in h_{S}^{1}, s_{\beta} \in h_{S}^{2}}\left\{s_{\alpha+\beta}\right\}, \quad\left[r_{1}^{L}+r_{2}^{L}-r_{1}^{L} \cdot r_{2}^{L}\right.$, $\left.r_{1}^{U}+r_{2}^{U}-r_{1}^{U} \cdot r_{2}^{U}\right]>$;

(2) $e_{1} \otimes e_{2}=<U_{s_{\alpha} \in h_{s}^{1}, s_{\beta} \in h_{S}^{2}}\left\{s_{\alpha \beta}\right\},\left[r_{1}^{L} \cdot r_{2}^{L}, r_{1}^{U} \cdot r_{2}^{U}\right]>$;
(3) $\lambda e=<U_{s_{\theta} \in h_{S}}\left\{s_{\lambda \theta}\right\},\left[1-\left(1-r^{L}\right)^{\lambda}, 1-\left(1-r^{R}\right)^{\lambda}\right]>$;

(4) $e^{\lambda}=<U_{s_{\theta} \in h_{S}}\left\{s_{\theta^{\lambda}}\right\},\left[\left(r^{L}\right)^{\lambda},\left(r^{U}\right)^{\lambda}\right]>$.

Example 1. Let $e=<\left\{s_{2}, s_{3}\right\},[0.4,0.6]>, e_{1}=$ $<\left\{s_{1}, s_{2}\right\},[0.2,0.6]>, e_{2}=<\left\{s_{3}, s_{4}, s_{5}\right\}, \quad[0.4,0.8]>$ be three IV2THFLEs and $\lambda=2$. Then,

(1) $e_{1} \oplus e_{2}=<\left\{s_{4}, s_{5}, s_{6}, s_{7}\right\},[0.52,0.92]>$;

(2) $e_{1} \otimes e_{2}=<\left\{s_{3}, s_{4}, s_{5}, s_{6}, s_{8}, s_{10}\right\},[0.08,0.48]>$;

(3) $\lambda e=<\left\{s_{4}, s_{6}\right\},[0.64,0.84]>$;

(4) $e^{\lambda}=<\left\{s_{4}, s_{9}\right\},[0.16,0.36]>$.

\subsection{Comparison laws of IV2THFLEs}

Note that an IV2THFLE consists of a HFLE and an interval number. The HFLE denotes DMs' linguistic evaluation interval index, while the interval number denotes the possible membership degree that an object belongs to the HFLE. For convenience, we transform both the HFLE and the interval membership degree into linguistic preference values. Then, we compare the IV2THFLEs by comparing these linguistic preference values.

Definition 3. Let $e_{1}=<h_{S}^{1}, \tilde{I}\left(e_{1}\right)>$ and $e_{2}=$ $<h_{S}^{2}, \tilde{I}\left(e_{2}\right)>$ be two IV2THFLEs with $\tilde{I}\left(e_{1}\right)=$ $\left[r_{1}^{L}, r_{1}^{U}\right], \quad \tilde{I}\left(e_{2}\right)=\left[r_{2}^{L}, r_{2}^{U}\right]$. Let $\operatorname{env}\left(h_{S}^{1}\right)$ and $\operatorname{env}\left(h_{S}^{2}\right)$ be the envelops of $h_{S}^{1}$ and $h_{S}^{2}$, respectively, where $\operatorname{env}\left(h_{S}^{1}\right)=\left[s_{\alpha_{1}}, s_{\alpha_{2}}\right] \quad$ and $\operatorname{env}\left(h_{S}^{2}\right)=\left[s_{\beta_{1}}, s_{\beta_{2}}\right]$. For convenience, $L P_{e_{1}}=$ $\left[\mu_{1}, \mu_{2}\right]=\left[\alpha_{1} \cdot r_{1}^{L}, \alpha_{2} \cdot r_{1}^{U}\right]$ and $L P_{e_{2}}=\left[\delta_{1}, \delta_{2}\right]=$ $\left[\beta_{1} \cdot r_{2}^{L}, \beta_{2} \cdot r_{2}^{U}\right]$ are called the linguistic preference values. The possibility degree of $e_{1} \geq e_{2}$ can be defined as:

$$
p\left(e_{1} \geq e_{2}\right)=\max \left\{1-\max \left(\frac{\delta_{2}-\mu_{1}}{l_{e_{1}}+l_{e_{2}}}, 0\right), 0\right\}
$$

where $l_{e_{1}}=\mu_{2}-\mu_{1}, l_{e_{2}}=\delta_{2}-\delta_{1}$.

According to Definition 3, we obtain

(1) $0 \leq p\left(e_{1} \geq e_{2}\right) \leq 1$;

(2) $p\left(e_{1} \geq e_{2}\right)+p\left(e_{2} \geq e_{1}\right)=1$;

(3) $p\left(e_{1} \geq e_{1}\right)=0.5$. 
To rank the IV2THFLEs $e_{i}(i=1, \ldots, m)$, we transform them into corresponding linguistic preference values $L P_{i}(i=1,2, \ldots, m)$, and then compare $L P_{i}(i=1,2, \ldots, m)$ by Eq. (6). Let $p_{i j}=$ $p\left(L P_{i} \geq L P_{j}\right)$. Then, a complementary matrix $P=\left(p_{i j}\right)_{m \times m}$ can be constructed with $p_{i j} \geq 0$, $p_{i j}+p_{j i}=1, \quad p_{i i}=0.5, \quad i=1,2, \ldots, m$. Summing all elements in each line of $P$, we have $p_{i}=\sum_{j=1}^{m} p_{i j}$. Finally, we can rank the IV2THELEs in descending order of the values of $p_{i}(i=1,2, \ldots, m)$.

Example 2. Let $e_{1}=\left\langle\left\{s_{2}, s_{3}\right\},[0.4,0.6]\right\rangle, \quad e_{2}=$ $\left\langle\left\{s_{1}, \mathrm{~s}_{2}\right\},[0.2,0.6]\right\rangle, e_{3}=\left\langle\left\{s_{3}, s_{4}, s_{5}\right\},[0.4,0.8]\right\rangle$ and $e_{4}$ $=\left\langle\left\{s_{3}, s_{4}\right\},[0.5,0.8]\right\rangle$ be four IV2THFLEs. A complementary matrix can be obtained as:

$$
P=\left[\begin{array}{cccc}
0.50 & 0.80 & 0.16 & 0.11 \\
0.20 & 0.50 & 0 & 0 \\
0.84 & 1.00 & 0.50 & 0.56 \\
0.89 & 1.00 & 0.44 & 0.50
\end{array}\right]
$$

Summing all values in each line of $P$, we have $p_{1}=1.57, p_{2}=0.70, p_{3}=2.90, p_{4}=2.83$. Since $p_{3} \succ p_{4} \succ p_{1} \succ p_{2}$, we have $e_{3} \succ e_{4} \succ e_{1} \succ e_{2}$.

\section{Aggregation operators for IV2THFLEs}

To solve various MADM problems under the IV2THFL environment, we define some aggregation operators of IV2THFLEs to obtain the overall linguistic aggregation information. In addition, a weighting method is proposed to overcome the instability of subjective weights. Finally, we develop a method to solve the MADM problems with IV2THFLEs.

\subsection{The IV2THFLWA, IV2THFLOWA, and} IV2THFLHA operators

Definition 4. Let $e_{i}(i=1,2, \ldots, n)$ be a collection of IV2THFLEs. Then an IV2THFLWA operator can be defined as:

$$
\operatorname{IV} 2 \operatorname{THFLWA}\left(e_{1}, e_{2}, \ldots, e_{n}\right)=\bigoplus_{i=1}^{n}\left(\omega_{i} e_{i}\right)
$$

where $\omega=\left(\omega_{1}, \omega_{2}, \ldots, \omega_{n}\right)^{T}$ is the weight vector of $e_{i}(i=1,2, \ldots, n)$ with $\omega_{i}>0, \quad \sum_{i=1}^{n} \omega_{i}=1$.

According to Definition 2, Theorem 1 can be derived.

Theorem 1. The aggregated result obtained by the IV2THFLWA operator is also an IV2THFLE, and

$$
\begin{gathered}
\text { IV 2THFLWA } \\
\left\{s_{\sum_{i=1}^{n} \omega_{i} \alpha_{i}}\right\},\left[1-\prod_{i=1}^{n}\left(1-r_{i}^{L}, \ldots, e_{n}\right)^{\omega_{i}}, 1-\prod_{i=1}^{n}\left(1-r_{s_{1} \in h_{s}^{1}, s_{\alpha_{2}} \in h_{S}^{2}, \ldots, s_{\alpha_{n}} \in h_{s}^{n}}^{\omega_{i}}\right]>\quad(8)\right.
\end{gathered}
$$

Proof. It can be proved by the mathematical induction on $n$.

(1) For $n=2$. Since

$$
\begin{aligned}
& \omega_{1} e_{1}=<\omega_{1} h_{S}^{1},\left[1-\left(1-r_{1}^{L}\right)^{\omega_{1}}, 1-\left(1-r_{1}^{U}\right)^{\omega_{1}}\right]> \\
& \omega_{2} e_{2}=<\omega_{2} h_{S}^{2},\left[1-\left(1-r_{2}^{L}\right)^{\omega_{2}}, 1-\left(1-r_{2}^{U}\right)^{\omega_{2}}\right]>
\end{aligned}
$$

Then

$$
\begin{aligned}
& \quad I V 2 \text { THFLWA } \omega_{\omega}\left(e_{1}, e_{2}\right)= \\
& \quad<U_{s_{\alpha_{1}} \in h_{s}^{1}, s_{\alpha_{2}} \in h_{S}^{2}}\left\{s_{\omega_{1} \cdot \alpha_{1}+\omega_{2} \cdot \alpha_{2}}\right\},\left[M_{1}, N_{1}\right]>=<U_{s_{a_{1}} \in h_{S}^{1}}, \\
& s_{s_{2} \in h_{S}^{2}}\left\{s_{\omega_{1} \cdot a_{1}+\omega_{2} \cdot a_{2}}\right\}, \quad\left[1-\left(1-r_{1}^{L}\right)^{\omega_{1}} \cdot\left(1-r_{2}^{L}\right)^{\omega_{2}},\right. \\
& \left.\left.\left(1-r_{1}^{U}\right)^{\omega_{1}} \cdot\left(1-r_{2}^{U}\right)^{\omega_{2}}\right]\right) \\
& \text { where } \quad M_{1}=2-\left(1-r_{1}^{L}\right)^{\omega_{1}} \quad-\quad\left(1-r_{2}^{L}\right)^{\omega_{2}}- \\
& \left(1-\left(1-r_{1}^{L}\right)^{\omega_{1}}\right) \cdot\left(1-\left(1-r_{2}^{L}\right)^{\omega_{1}}\right), N_{1}=2-\left(1-r_{1}^{U}\right)^{\omega_{1}}- \\
& \left(1-r_{2}^{U}\right)^{\omega_{2}}-\left(1-\left(1-r_{1}^{U}\right)^{\omega_{1}}\right) \cdot\left(1-\left(1-r_{2}^{U}\right)^{\omega_{2}}\right) .
\end{aligned}
$$

(2) If Eq. (8) holds for $n=k$, that is IV2THFLWA $\left(e_{1}, e_{2}, \ldots, e_{k}\right)=<\cup_{s_{\alpha_{1}} \in h_{s}^{1}}$, $s_{\alpha_{2} \in h_{S}^{2}, \ldots, s_{\alpha_{k}} \in h_{S}^{k}}\left\{s_{\sum_{i=1}^{k} \omega_{i} \alpha_{i}}\right\}, \quad\left[1-\prod_{i=1}^{k}\left(1-r_{i}^{L}\right)^{\sigma_{i}}, 1-\right.$ $\left.1-\prod_{i=1}^{k}\left(1-r_{i}^{U}\right)^{\omega_{i}}\right]>$

Then, when $n=k+1$, by the operations in Definition 2, we have

$$
\text { IV2THFLWA } \operatorname{Ti}_{\omega}\left(e_{1}, e_{2}, \ldots, e_{k+1}\right)=\left\langle\cup_{s_{\alpha_{1}} \in h_{s}^{1}, s_{\alpha_{2}} \in h_{s}^{2}},\right.
$$$$
\ldots . s_{\alpha_{k+1}} \in h_{s}^{k+1}\left\{s_{\sum_{i=1}^{k} \omega_{i} \alpha_{i}+\omega_{k+1} \alpha_{k+1}}\right\},\left[M_{2}, N_{2}\right]>=\left\langle\cup_{s_{\alpha_{1}} \in h_{s}^{1}},\right.
$$$$
s_{\alpha_{2}} \in h_{S}^{2}, \ldots, s_{\alpha_{k+1}} \in h_{S}^{k+1}\left\{s_{\sum_{i=1}^{k+1} \omega_{i} \alpha_{i}}\right\}, \quad\left[1-\prod_{i=1}^{k+1}\left(1-r_{i}^{L}\right)^{\omega_{i}}, \quad 1-\right.
$$

$\left.\prod_{i=1}^{k+1}\left(1-r_{i}^{U}\right)^{\omega_{i}}\right]>$ 
where $\quad M_{2}=2-\prod_{i=1}^{k}\left(1-r_{i}^{L}\right)^{\omega_{i}}-\left(1-r_{k+1}^{L}\right)^{\omega_{k+1}}-$ $\left(1-\prod_{i=1}^{k}\left(1-r_{i}^{L}\right)^{\omega_{i}}\right) \cdot\left(1-\left(1-r_{k+1}^{L}\right)^{\omega_{k+1}}\right), \quad N_{2}=2-$ $\prod_{i=1}^{k}\left(1-r_{i}^{U}\right)^{\omega_{i}}-\left(1-r_{k+1}^{U}\right)^{\omega_{1}}-\left(1-\prod_{i=1}^{k}\left(1-r_{i}^{U}\right)^{\omega_{i}}\right)$. $\left(1-\left(1-r_{k+1}^{U}\right)^{\omega_{k+1}}\right)$.

Thus, Eq. (8) holds for $n=k+1$. Therefore, Eq. (8) holds for all $n$.

Inspired by the ordered weighted averaging (OWA) operator [32], we define the IV2THFLOWA operator according to the OWA operator.

Definition 5. Let $e_{i}(i=1,2, \ldots, n)$ be a collection of IV2THFLEs, $e_{\sigma(i)}$ be the $i$ th largest of them, $\omega=\left(\omega_{1}, \omega_{2}, \ldots, \omega_{n}\right)^{T}$ be the aggregation associated vector such that $\omega_{i} \in[0,1]$ and $\sum_{i=1}^{n} \omega_{i}=1$. Then an IV2THFLOWA operator is defined as:

$$
\text { IV2THFLOWA }\left(e_{1}, e_{2}, \ldots, e_{n}\right)=\bigoplus_{i=1}^{n}\left(\omega_{i} e_{\sigma(i)}\right)
$$

Theorem 2. The aggregated result obtained by the IV2THFLOWA operator is also an IV2THFLE, and IV2THFLOWA $\left(e_{1}, e_{2}, \ldots, e_{n}\right)$

$=<\cup_{s_{\alpha_{\sigma(1)}} \in h_{S}^{\sigma(1)}, s_{\alpha_{\sigma(2)}} \in h_{S}^{\sigma(2)}, \ldots, s_{\alpha_{\sigma(n)}} \in h_{S}^{\sigma(n)}}\left\{s_{\sum_{i=1}^{n} \omega_{i} \alpha_{\sigma(i)}}\right\}$,

$\left[1-\prod_{i=1}^{n}\left(1-r_{\sigma(i)}^{L}\right)^{\omega_{i}}, 1-\prod_{i=1}^{n}\left(1-r_{\sigma(i)}^{U}\right)^{\omega_{i}}\right]>$

The proof of Theorem 2 is similar to that of Theorem 1.

Definition 6. Let $e_{i}(i=1,2, \ldots, n)$ be a set of IV2THFLEs, $\quad w=\left(w_{1}, w_{2}, \ldots, w_{n}\right)^{T} \quad$ be the aggregation associated vector such that $w_{i} \in[0,1]$ and $\sum_{i=1}^{n} w_{i}=1 . n$ is the balancing coefficient. Then we define an IV2THFL hybrid averaging (IV2THFLHA) operator as follows:

$$
\text { IV 2THFLHA } A_{\omega w}\left(e_{1}, e_{2}, \ldots, e_{n}\right)=\bigoplus_{i=1}^{n}\left(\omega_{i} \vec{e}_{\sigma(i)}\right)
$$

where $\omega=\left(\omega_{1}, \omega_{2}, \ldots, \omega_{n}\right)^{T}$ is the aggregation associated vector, with $\omega_{i} \in[0,1], \sum_{i=1}^{n} \omega_{i}=1$, and $\vec{e}_{\sigma(i)}$ is the $i$ th largest element of $\vec{e}_{i}\left(\vec{e}_{i}=n w_{i} e_{i}, i=1,2, \ldots, n\right) \quad . \quad$ Especially, if $\omega=(1 / n, 1 / n, \ldots, 1 / n)^{T}$ and $w=(1 / n, 1 / n, \ldots, 1 / n)^{T}$, then the IVHF2TLHA operator is reduced to the IV2THFLWA operator and IV2THFLOWA operator, respectively.

Theorem 3. The aggregated result obtained by the IV2THFLHA operator is also an IV2THFLE, and

IV2THFLHA $A_{\omega w}\left(e_{1}, e_{2}, \ldots, e_{n}\right)$

$=<\bigcup_{s_{\vec{\alpha}_{\sigma(1)}} \in \vec{h}_{S}^{\sigma(1)}, s_{\vec{\alpha}_{\sigma(2)}} \vec{h}_{S}^{\sigma(2)}, \ldots, s_{\vec{\alpha}_{\sigma(2)}} \in \vec{h}_{S}^{\sigma(n)}}\left\{s_{\sum_{i=1}^{n} \omega_{i} \vec{\alpha}_{\sigma(i)}}\right\}$,

$\left[1-\prod_{i=1}^{n}\left(1-\vec{r}_{\sigma(i)}^{L}\right)^{\omega_{i}}, 1-\prod_{i=1}^{n}\left(1-\vec{r}_{\sigma(i)}^{U}\right)^{\omega_{i}}\right]>$

The proof of Theorem 3 is similar to that of Theorem 1.

\subsection{The GIV2THFLWA, GIV2THFLOWA, GIV2THFLHA operators}

In this subsection, we define some generalized operators under the IV2THFL environment.

Definition 7. Let $e_{i}(i=1,2, \ldots, n)$ be a set of IV2THFLEs, and $\omega=\left(\omega_{1}, \omega_{2}, \ldots, \omega_{n}\right)^{T}$ be the weight vector of $e_{i}(i=1,2, \ldots, n)$ with $\omega_{i} \in[0,1]$, $\sum_{i=1}^{n} \omega_{i}=1$ and $\lambda>0$. Then a GIV2THFLWA operator is a mapping GIV2THFLWA: $e^{n} \rightarrow e$, where

$$
\operatorname{GIV} 2 \operatorname{THFLWA} A_{\lambda}\left(e_{1}, e_{2}, \ldots, e_{n}\right)=\left(\bigoplus_{i=1}^{n}\left(\omega_{i} e_{i}^{\lambda}\right)\right)^{1 / \lambda}
$$

Theorem 4. The aggregated result by the GIV2THFLWA operator is also an IV2THFLE, and GIV2THFLWA $\left(e_{1}, e_{2}, \ldots, e_{n}\right)=<\cup_{s_{\alpha_{1}} \in h_{S}^{1}, s_{\alpha_{2}} \in h_{S}^{2}, \ldots, s_{\alpha_{n}} \in h_{S}^{n}}$

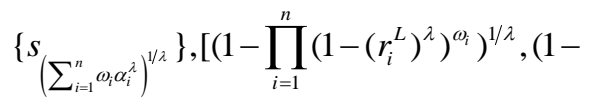

$\left.\left.\prod_{i=1}^{n}\left(1-\left(r_{i}^{U}\right)^{\lambda}\right)^{\omega_{i}}\right)^{1 / \lambda}\right]>$

Proof. (1) For $n=2$. Since 
$\omega_{1} e_{1}^{\lambda}=<\omega_{1}\left(h_{s}^{1}\right)^{\lambda},\left[1-\left(1-\left(r_{1}^{L}\right)^{\lambda}\right)^{\omega_{1}}, 1-\left(1-\left(r_{1}^{U}\right)^{\lambda}\right)^{\omega_{1}}\right]>$

$\omega_{2} e_{2}^{\lambda}=<\omega_{2}\left(h_{S}^{2}\right)^{\lambda},\left[1-\left(1-\left(r_{2}^{L}\right)^{\lambda}\right)^{\omega_{2}}, 1-\left(1-\left(r_{2}^{U}\right)^{\lambda}\right)^{\omega_{2}}\right]>$

Then

$\operatorname{GIV} 2 \operatorname{THFLWA}\left(e_{1}, e_{2}\right)=$

$<\cup_{s_{\alpha_{1}} \in h_{s}^{1}, s_{\alpha_{2}} \in h_{S}^{2}}\left\{s_{\left(\omega_{1}\left(\alpha_{1}\right)^{2}+\omega_{2}\left(\alpha_{2}\right)^{2}\right)^{1 / \lambda}}\right\},\left[M_{3}, N_{3}\right]>$

$=<\cup_{s_{\alpha_{1}} \in h_{S}^{1}, s_{\alpha_{2}} \in h_{S}^{2}}\left\{s\left(\sum_{i=1}^{2} \omega_{i}\left(\alpha_{i}\right)^{\lambda}\right)^{1 / \lambda}\right\}$,

$\left[\left(1-\prod_{i=1}^{2}\left(1-\left(r_{i}^{L}\right)^{\lambda}\right)^{\omega_{i}}\right)^{1 / \lambda},\left(1-\prod_{i=1}^{2}\left(1-\left(r_{i}^{U}\right)^{\lambda}\right)^{\omega_{i}}\right)^{1 / \lambda}\right]>$

where $\quad M_{3}=\left(2-\left(1-\left(r_{1}^{L}\right)^{\lambda}\right)^{\omega_{1}}-\left(1-\left(r_{2}^{L}\right)^{\lambda}\right)^{\omega_{2}}-\right.$

$\left.\left(1-1-\left(r_{1}^{L}\right)^{\lambda}\right)^{\omega_{1}} \cdot\left(1-\left(r_{2}^{L}\right)^{\lambda}\right)^{\omega_{2}}\right)^{1 / \lambda} \quad, \quad N_{3}=(2-$

$\left(1-\left(r_{1}^{U}\right)^{\lambda}\right)^{\omega_{1}}-\left(1-\left(r_{2}^{U}\right)^{\lambda}\right)^{\omega_{2}}-\left(1-1-\left(r_{1}^{U}\right)^{\lambda}\right)^{\omega_{1}}$.

$\left.\left(1-\left(r_{2}^{U}\right)^{\lambda}\right)^{\omega_{2}}\right)^{1 / \lambda}$.

(2) If Eq. (14) holds for $n=k$, that is

$\operatorname{GIV} 2 \operatorname{THFLWA} A_{\omega}\left(e_{1}, e_{2}, \ldots, e_{k}\right)=<\cup_{s_{\alpha_{1}} \in h_{s}^{1}, s_{\alpha_{2}} \in h_{s}^{2}}$,

$\ldots, s_{\alpha_{k}} \in h_{s}^{k}\left\{s\left(\sum_{i=1}^{k} \omega_{i} \alpha_{i}^{\lambda}\right)^{1 / \lambda}\right\},\left[\left(1-\prod_{i=1}^{k}\left(1-\left(r_{i}^{L}\right)^{\lambda}\right)^{\omega_{i}}\right)^{1 / \lambda}\right.$,

$\left.\left(1-\prod_{i=1}^{k}\left(1-\left(r_{i}^{U}\right)^{\lambda}\right)^{\omega_{i}}\right)^{1 / \lambda}\right]>$

Then, when $n=k+1$, we have

$\operatorname{GIV} 2$ THFLWA $A_{\omega}\left(e_{1}, e_{2}, \ldots, e_{k+1}\right)=<\cup_{s_{\alpha_{1}} \in h_{s}^{1}, s_{\alpha_{2}} \in h_{s}^{2}}$,

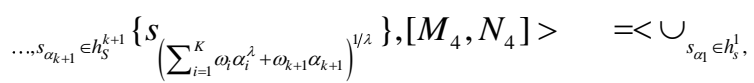

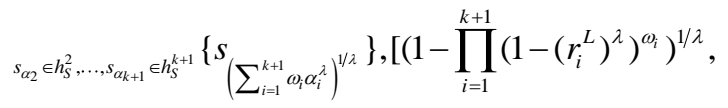

$\left.\left(1-\prod_{i=1}^{k+1}\left(1-\left(r_{i}^{U}\right)^{\lambda}\right)^{\omega_{i}}\right)^{1 / \lambda}\right]>$

where $M_{4}=\left(2-\prod_{i=1}^{k}\left(1-\left(r_{i}^{L}\right)^{\lambda}\right)^{\omega_{i}}-\left(1-\left(r_{k+1}^{L}\right)^{\lambda}\right)^{\omega_{K+1}}-\right.$ $\left(1-\prod_{i=1}^{k}\left(1-\left(r_{i}^{L}\right)^{\lambda}\right)^{\omega_{i}}\right) \cdot\left(1-\left(1-\left(r_{K+1}^{L}\right)^{\lambda}\right)^{\omega_{1}}\right)^{1 / \lambda} \quad, \quad N_{4}=$ $\left(2-\prod_{i=1}^{k}\left(1-\left(r_{i}^{U}\right)^{\lambda}\right)^{\omega_{i}} \quad-\quad\left(1-\left(r_{k+1}^{U}\right)^{\lambda}\right)^{\omega_{k+1}} \quad-\right.$ $\left(1-\prod_{i=1}^{k}\left(1-\left(r_{i}^{U}\right)^{\lambda}\right)^{\omega_{i}}\right) \cdot\left(1-\left(1-\left(r_{K+1}^{U}\right)^{\lambda}\right)^{\omega_{1}}\right)^{1 / \lambda}$.

Thus, Eq. (14) holds for $n=k+1$. Hence, Eq. (14) holds for all $n$.

Definition 8. Let $e_{i}(i=1,2, \ldots, n)$ be a collection of
IV2THFLEs, $e_{\sigma(i)}$ be the $i$ th largest of them, $\omega=\left(\omega_{1}, \omega_{2}, \ldots, \omega_{n}\right)^{T}$ be the aggregation associated vector such that $\omega_{i} \in[0,1]$ and $\sum_{i=1}^{n} \omega_{i}=1$. Then a GIV2THFLOWA operator is defined as:

$\operatorname{GIV} 2$ THFLOWA $\lambda_{\lambda}\left(e_{1}, e_{2}, \ldots, e_{n}\right)=\left(\bigoplus_{i=1}^{n}\left(\omega_{i} e_{\sigma(i)}^{\lambda}\right)\right)^{1 / \lambda}$

Theorem 5. The aggregated value of the GIV2THFLOWA operator is also an IV2THFLE, and GIV2THFLOWA $A_{\omega}\left(e_{1}, e_{2}, \ldots, e_{n}\right)=<\bigcup_{s_{\alpha_{\sigma(1)}} \in h_{s}^{\sigma(1)}, s_{\alpha_{\sigma(2)}} \in h_{S}^{\sigma(2)},}$

$\ldots . s_{\alpha_{\sigma(n)}} \in h_{s}^{\sigma(n)}\left\{s\left(\sum_{i=1}^{n} \omega_{i} \alpha_{\sigma(i)}^{\lambda}\right)^{1 / \lambda}\right\},\left[\left(1-\prod_{i=1}^{n}\left(1-r_{\sigma(i)}^{L}\right)^{\omega_{i}}\right)^{1 / \lambda}\right.$,

$\left.\left(1-\prod_{i=1}^{n}\left(1-r_{\sigma(i)}^{U}\right)^{\omega_{i}}\right)^{1 / \lambda}\right]>$

The proof of Theorem 5 is similar to that of Theorem 4.

Definition 9. Let $e_{i}(i=1,2, \ldots, n)$ be a set of IV2THFLEs, $\quad w=\left(w_{1}, w_{2}, \ldots, w_{n}\right)^{T}$ be the aggregation associated vector such that $w_{i} \in[0,1]$ and $\sum_{i=1}^{n} w_{i}=1 . n$ is the balancing coefficient. Then a GIV2THFLHA operator is defined as:

$\operatorname{GIV} 2 \operatorname{THFLHA} A_{\lambda}\left(e_{1}, e_{2}, \ldots, e_{n}\right)=\left(\bigoplus_{i=1}^{n}\left(\omega_{i} \vec{e}_{\sigma(i)}^{\lambda}\right)\right)^{1 / \lambda}$

where $\omega=\left(\omega_{1}, \omega_{2}, \ldots, \omega_{n}\right)^{T}$ is the aggregation associated vector with $\omega_{i} \in[0,1], \sum_{i=1}^{n} \omega_{i}=1$, and $\vec{e}_{\sigma(i)}$ is the $i$ th largest element of $\vec{e}_{i}\left(\vec{e}_{i}=n w_{i} e_{i}, i=1,2, \ldots, n\right)$.

Theorem 6. The aggregated result of the GIV2THFLHA operator is also an IV2THFLE, and

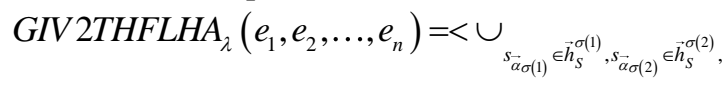

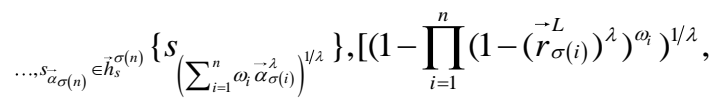
$\left.\left(1-\prod_{i=1}^{n}\left(1-\left(\vec{r}_{\sigma(i)}^{U}\right)^{\lambda}\right)^{\omega_{i}}\right)^{1 / \lambda}\right]>$

The proof of Theorem 6 is similar to that of Theorem 4. 


\subsection{A method to determine the weights of attributes}

The subjective weights of attributes lead to the instability of decision results. To overcome this problem, in this subsection, we propose a method to obtain the weights of attributes under the IV2THFL environment.

The differences of the evaluation information between attributes have an influence on the accuracy of decision results. The smaller the differences are, the more precise the decision results would be. To compute the difference between two IVHFEs, Chen et al. [33] defined the distance measures for the IVHFEs. Motivated by this idea, we can defined the distance measures for IV2THFLEs. We first transform the evaluation information of alternative $A_{i}$ with respect to attribute $C_{j}$ into the linguistic preference value $L P_{i j}$ based on Definition 3. Then we obtain the subscript of $L P_{i j}$, i.e., $\operatorname{sub}\left(L P_{i j}\right)=$ $\left[L P_{i j}^{L}, L P_{i j}^{U}\right]$. We compute the difference between attributes $C_{l}$ and $C_{k}$ using the distance measure proposed in Ref. [33] and thus obtain

$$
\begin{aligned}
D_{l k}= & d\left(L P_{i l}, L P_{i k}\right)=\frac{1}{2 m} \sum_{i=1}^{m}\left(\left|L P_{i l}^{L}-L P_{i k}^{L}\right|+\right. \\
& \left.\left|L P_{i l}^{U}-L P_{i k}^{U}\right|\right) \quad(i=1,2, \ldots m ; l, k=1,2, \ldots n)
\end{aligned}
$$

Let $D_{l}=\sum_{k=1}^{m} D_{l k}$ be the deviation of attribute $C_{l}$ from the remaining attributes. The smaller $D_{l}$ is, the closer attribute $C_{l}$ is to that of the rest attributes, and hence the more valuable evaluation information of attribute $C_{l}$ provides. Thus $C_{l}$ should be assigned a large weight. The weights can be calculated as:

$$
\omega_{l}=\frac{\left(D_{l}\right)^{-1}}{\sum_{l}^{n}\left(D_{l}\right)^{-1}} \quad(l=1,2, \ldots, n)
$$

\subsection{A method for MADM with IV2THFLES}

For a MADM problem with uncertain linguistic information, let $A=\left\{A_{1}, A_{2}, \ldots, A_{m}\right\}$ be a discrete collection of variables, $C=\left\{C_{1}, C_{2}, \ldots, C_{n}\right\}$ be a discrete collection of attributes, whose weight vector is $\omega=\left(\omega_{1}, \omega_{2}, \ldots, \omega_{n}\right)^{T}$ with $\omega_{j} \geq 0, j=1,2, \ldots n$, $\sum_{j=1}^{n} \omega_{j}=1$. Suppose that $T=\left(t_{i j}\right)_{m \times n}$ is the decision matrix, where $t_{i j}$ is the preference information in the form of IV2THFLE. In the following, the GIV2THFLWA operator is used to develop a method to solve the MADM problems under the IV2THFL environment.

$$
\begin{aligned}
& \text { Algorithm } 1 \\
& \text { Step 1. Compute the difference between any two } \\
& \text { attributes } C_{l} \text { and } C_{k} \text { and then determine the } \\
& \text { weights of attributes by Eq. (20). } \\
& \text { Step 2. Utilize the GIV2THFLWA operator to } \\
& \text { aggregate the evaluation arguments in the decision } \\
& \text { matrix } T \text {, and obtain the overall IV2THFL } \\
& \text { preference values } t_{i}(i=1,2, \ldots m) \text { of the alternative } \\
& A_{i} \text {. We have } \\
& \qquad t_{i}=G I V 2 T H F L W A_{\lambda}\left(t_{i 1}, t_{i 2}, \ldots, t_{i n}\right)=\left(\bigoplus_{i=1}^{n}\left(\omega_{j} t_{i j}^{\lambda}\right)\right)^{1 / \lambda} \\
& \qquad(i=1,2, \ldots m)
\end{aligned}
$$

Step 3. Transform the collective preference information $t_{i}(i=1,2, \ldots m)$ into the corresponding linguistic preference values $L P_{i}(i=1,2, \ldots, m)$ based on Definition 3.

Step 4. Compare each value $L P_{i}$ with all values of $L P_{j}(j=1,2, \ldots, m)$ by Definition 3. For simplicity, a complementary matrix $P=\left(p_{i j}\right)_{m \times n}$ is constructed, where $p_{i j} \geq 0, p_{i j}+p_{j i}=1, p_{i i}=0.5$. Summing all the values in each line of $P$, we have $p_{i}=\sum_{j=1}^{n} p_{i j}(i=1,2, \ldots, m)$.

Step 5. Select the best alternative according to the values of $p_{i}(i=1,2, \ldots, m)$.

In Algorithm 1, Step 1 is to obtain the objective weights according to the differences of attributes. Step 2 is to derive the collective IV2THFL preference values of alternatives by the GIV2THFLWA operator. Step 3 is to transform the overall preference information into the corresponding linguistic preference values. Step 4 is to compare the linguistic preference values and establish the complementary matrix. Step 5 is to rank all the alternatives according to the values of each line of the complementary matrix. 


\section{Case study: Global mineral investment evaluation}

In this section, we apply the proposed MADM method in a practical example concerning the global mineral investment evaluation (adapted from Ref. [34]). Then the proposed method is compared with other existing methods.

\subsection{Case description}

ABC Nonferrous Metals Co. Ltd. is a large state-owned company whose main business is producing and selling nonferrous metals. The company evaluates the global mineral investment business according to the oversea investment department, which consists of executive managers and several experts in this field. Recently, this department decided to select several alternatives from some foreign countries based on preliminary survey. After detailed analysis, four countries $\left\{A_{1}, A_{2}, A_{3}, A_{4}\right\}$ are taken into consideration. Three factors are finally considered, including $C_{1}$ : resources, $C_{2}$ : politics and policy and $C_{3}$ : infrastructure.

To obtain the decision information, the LTS $S=\left\{s_{0}\right.$ : nothing, $s_{1}$ :very low, $s_{2}$ :low, $s_{3}$ :medium, $s_{4}:$ high, $s_{5}$ :very high, $s_{6}:$ perfect $\}$ is used. The decision information takes the form of IV2THFLEs where $C_{j}\left(A_{i}\right)$ is the evaluation argument of alternative $A_{i}$ on criterion $C_{j}$. In $C_{j}\left(A_{i}\right)$ there is a consensus on the chosen LTS and each DM can use a value to express his/her opinions, i.e., the value $C_{j}\left(A_{i}\right)$ denotes to what degree $A_{i}$ matches this given linguistic terms under $C_{j}$. DMs gave their own evaluation values in the form of IV2THFLEs based on the survey of these four countries as well as their knowledge and experience. Consequently, following a heated discussion, they came to a consensus on the final decision as shown in decision matrix $T$.

$$
\begin{aligned}
& \begin{array}{lll}
C_{1} & C_{2} & C_{3}
\end{array}
\end{aligned}
$$

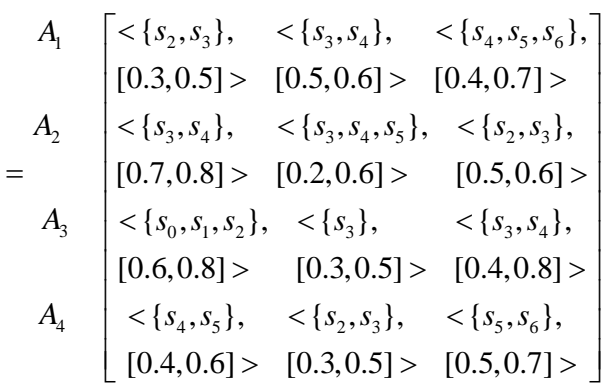

\subsection{Application of the proposed method}

To overcome the instability of subjective weights, the weights of criteria are calculated by Eq. (20) and we obtain $\omega_{1}=0.34, \omega_{2}=0.36$ and $\omega_{3}=0.29$. To get the best alternative, we let $\lambda=0.1$ and utilize the GIV2THFLWA operator to aggregate all the linguistic evaluation information $t_{i j}(i=1,2,3,4$, $j=1,2,3)$ in decision matrix $T$. Then we can obtain the overall preference values $t_{i}(i=1,2,3,4)$ as $t_{1}=\left\langle\left\{s_{2.58}, s_{2.87}, s_{2.95}, s_{3.28}, s_{2.76}, s_{3.07}, s_{3.16}, s_{3.51}\right.\right.$, $\left.\left.s_{2.92}, s_{3.24}, s_{3.34}, s_{3.70}\right\},[0.39,0.59]\right\rangle, t_{2}=\left\langle\left\{s_{2.41}, s_{2.69}\right.\right.$, $\left.s_{2.92}, s_{2.67}, s_{2.97}, s_{3.23}, s_{2.71}, s_{3.02}, s_{3.28}, s_{3.00}, s_{3.33}, s_{3.61}\right\}$, $[0.47,0.67]>, \quad t_{3}=<\left\{s_{0.04}, s_{1.89}, s_{2.36}, s_{0.05}, s_{2.06}, s_{2.58}\right\}$, $[0.43,0.71]>, t_{4}=<\left\{s_{3.02}, s_{3.49}, s_{3.27}, s_{3.77}, s_{3.20}, s_{3.68}\right.$, $\left.s_{3.46}, s_{3.98}\right\}, \quad[0.38,0.59]>\quad$ Subsequently, we transform the overall preference values into their corresponding linguistic preference values as $L P_{1}=\left[s_{1.00}, s_{2.18}\right] \quad, \quad L P_{2}=\left[s_{1.13}, s_{2.42}\right] \quad$, $L P_{3}=\left[s_{0.02}, s_{1.83}\right], \quad L P_{4}=\left[s_{1.15}, s_{2.35}\right]$. According to Definition 3 and the linguistic preference values $L P_{i}(i=1,2,3,4)$, we obtain the complementary matrix $P$ as follows:

$$
P=\left[\begin{array}{llll}
0.50 & 0.43 & 0.72 & 0.43 \\
0.57 & 0.50 & 0.77 & 0.51 \\
0.28 & 0.23 & 0.50 & 0.23 \\
0.57 & 0.49 & 0.77 & 0.50
\end{array}\right]
$$

Then, we have $p_{1}=2.08, p_{2}=2.35, p_{3}=1.24$, $p_{4}=2.33$. We rank $p_{i}(i=1,2,3,4)$ and obtain $A_{2} \succ A_{4} \succ A_{1} \succ A_{3}$. Thus, the best alternative is $A_{2}$.

In the above example, we only give the ranking order according to the GIV2THFLWA operator with $\lambda=0.1$. As the parameter $\lambda$ changes, different 
results can be obtained, shown as Table 2. By Table 2, we can find that the decision results are highly related to $\lambda$. As $\lambda$ increases, $A_{2}$ is the best choice first, and then $A_{4}$ becomes the best choice in the case $\lambda \geq 0.5$. The DMs can choose the values of $\lambda$ according to their preferences.

Table 2. The ranking results with respect to different values of $\lambda$

\begin{tabular}{cccccc}
\hline & $L P_{1}$ & $L P_{2}$ & $L P_{3}$ & \multicolumn{1}{c}{$L P_{4}$} & \multicolumn{1}{c}{ Ranking } \\
\hline$\lambda=0.1$ & {$\left[s_{1.00}, s_{2.18}\right]$} & {$\left[s_{1.13}, s_{2.42}\right]$} & {$\left[s_{0.02}, s_{1.83}\right]$} & {$\left[s_{1.15}, s_{2.35}\right]$} & $A_{2} \succ A_{4} \succ A_{1} \succ A_{3}$ \\
$\lambda=0.2$ & {$\left[s_{1.08}, s_{2.32}\right]$} & {$\left[s_{1.20}, s_{2.57}\right]$} & {$\left[s_{0.15}, s_{1.93}\right]$} & {$\left[s_{1.24}, s_{2.48}\right]$} & $A_{2} \succ A_{4} \succ A_{1} \succ A_{3}$ \\
$\lambda=0.5$ & {$\left[s_{1.14}, s_{2.43}\right]$} & {$\left[s_{1.27}, s_{2.68}\right]$} & {$\left[s_{0.55}, s_{2.02}\right]$} & {$\left[s_{1.32}, s_{2.61}\right]$} & $A_{4} \succ A_{2} \succ A_{1} \succ A_{3}$ \\
$\lambda=1.0$ & {$\left[s_{1.18}, s_{2.52}\right]$} & {$\left[s_{1.34}, s_{2.74}\right]$} & {$\left[s_{0.87}, s_{2.09}\right]$} & {$\left[s_{1.40}, s_{2.70}\right]$} & $A_{4} \succ A_{2} \succ A_{1} \succ A_{3}$ \\
$\lambda=2.0$ & {$\left[s_{1.25}, s_{2.65}\right]$} & {$\left[s_{1.44}, s_{2.83}\right]$} & {$\left[s_{1.11}, s_{2.21}\right]$} & {$\left[s_{3.76}, s_{4.71}\right]$} & $A_{4} \succ A_{2} \succ A_{1} \succ A_{3}$ \\
$\lambda=5.0$ & {$\left[s_{1.43}, s_{3.00}\right]$} & {$\left[s_{1.66}, s_{3.05}\right]$} & {$\left[s_{1.37}, s_{2.46}\right]$} & {$\left[s_{1.76}, s_{3.13}\right]$} & $A_{4} \succ A_{2} \succ A_{1} \succ A_{3}$ \\
\hline
\end{tabular}

\subsection{Comparison analyses}

To illustrate the advantages, the presented method is compared with other two representative MADM methods.

\section{Case 1. Comparison with the MADM method with HFLTSs}

Rodríguez et al. [8] developed a MADM model with HFLTS, and utilized min-upper and max-lower operators to obtain the linguistic evaluation intervals. For comparison, we utilize the MADM method in Ref. [8] to solve the illustrative example above. To begin with, we obtain the product of the subscripts corresponding to linguistic terms and the discrete values within the interval-valued membership degrees. Then the IV2THFLEs are transformed into the HFLEs by integrating the possible interval membership degrees and each HFLTS. For example, an IV2THFLE $<\left\{s_{2}, s_{3}\right\},[0.3,0.5]>$ can be replaced by a HFLE $\left\{s_{0.6}, s_{0.8}, s_{1.0}, s_{0.9}, s_{1.2}, s_{1.5}\right\}$. The transformed decision matrix $T D$ under hesitant fuzzy linguistic environment is shown as follows:

$$
\left.\begin{array}{c}
C_{1} \\
A_{1}
\end{array} C_{2} \quad \begin{array}{lll}
\left\{s_{0.6}, s_{0.8}, s_{1.0},\right. & \left\{s_{1.5}, s_{1.8}, s_{2.0}\right. & \left\{s_{1.6}, s_{2.8}, s_{2.0},\right. \\
\left.s_{0.9}, s_{1.2}, s_{1.5}\right\} & \left., s_{2.4}\right\} & \left.s_{3.5}, s_{2.4}, s_{4.2}\right\} \\
\left\{s_{2.1}, s_{2.4}, s_{2.8},\right. & \left\{s_{0.6}, s_{1.8}, s_{0.8},\right. & \left\{s_{1.0}, s_{1.2}, s_{1.5},\right. \\
\left.s_{3.2}\right\} & \left.s_{2.4}, s_{1.0}, s_{3.0}\right\} & \left.s_{1.8}\right\} \\
\left\{s_{0}, s_{0.6}, s_{0.8},\right. & \left\{s_{0.9}, s_{1.5}\right\} & \left\{s_{1.2}, s_{2.4}, s_{1.6},\right. \\
\left.s_{1.2}, s_{1.6}\right\} & \left.s_{3.2}\right\} \\
\left\{s_{1.6}, s_{2.4}, s_{2.0},\right. & \left\{s_{0.6}, s_{1.0},\right. & \left\{s_{2.5}, s_{3.5}, s_{3.0}\right. \\
\left.s_{3.0}\right\} & \left.s_{0.9}, s_{1.5}\right\} & \left.s_{4.2}\right\}
\end{array}\right]
$$

According to the min-upper and max-lower
operators, we obtain the linguistic intervals $L I_{i}$ $(i=1,2,3,4) \quad$ as $\quad L I_{1}=\left[s_{1.5}, s_{1.6}\right], L I_{2}=\left[s_{1.8}, s_{2.1}\right]$, $L I_{3}=\left[s_{1.2}, s_{1.5}\right] \quad, \quad L I_{4}=\left[s_{1.5}, s_{2.5}\right] \quad$. Then, the nondominance degrees of alternatives are obtained as: $N D D_{1}=0, N D D_{2}=0.46, N D D_{3}=0, N D D_{4}=$ 0.92 . The ranking of the alternatives is: $A_{4} \succ A_{2} \succ A_{1}=A_{3}$. From Table 2, when $\lambda=0.5$, $1,2,5$, obviously, the most desirable alternative is consistent with that obtained by the proposed method. However, in other cases, the ranking results obtained by our method is inconsistent with that obtained by the method in Ref. [8]. The main reason is that the proposed method can effectively retain the completeness of decision information in operation process.

\section{Case 2. Comparison with the MADM method with HFULSs}

Lin et al. [23] proposed a MADM method based on HFLS. To compare it with the proposed method, we need to transform the IV2THFLEs into the HFLEs. We obtain the mean value of the subscripts corresponding to all linguistic terms in the IV2THFLEs, and then transform the interval membership degree into some discrete values in it. For example, an IV2THFLE $<\left\{s_{2}, s_{3}\right\}$, [0.3,0.5] $>$ can be replaced by a HFLE $<s_{2.5},(0.3,0.4,0.5)>$. By the approach in Ref. [23], we get $s\left(A_{1}\right)=1.8698$, $s\left(A_{2}\right)=1.9927, \quad s\left(A_{3}\right)=1.4858, \quad s\left(A_{4}\right)=2.1316$. Since $s\left(A_{4}\right)>s\left(A_{2}\right)>s\left(A_{1}\right)>s\left(A_{3}\right)$, the ranking of alternatives is $A_{4} \succ A_{2} \succ A_{1} \succ A_{3}$. Obviously, when $\lambda=0.5,1,2,5$, the above ranking is the same 
as that obtained by the proposed method. When $\lambda=0.1,0.2$, the above ranking is different from that obtained by the method in this paper. Thus, DMs can flexibly select the value of parameter $\lambda$ to make decisions according to their preferences.

Compared with the above methods within different contexts, the advantages of the proposed method for MADM problems under IV2THFL environment are listed as follows:

(1) The IV2THFLEs can provide a flexible choice for DMs and closely depict the precise membership degrees of a linguistic variable to HFLTS. The IV2THFLEs not only give the possible linguistic terms but also consider the possible membership degrees. In addition, the IV2THFLEs can retain the completeness of decision information, which are more precise than HFLTS.

(2) We define some generalized aggregation operators for IV2THFLEs. Different decision results can be obtained when different values of $\lambda$ are used. Thus, DMs can flexibly select the value of $\lambda$ according to their preferences.

(3) We propose a method to obtain the weights of attributes based on the differences of attributes, which avoids the instability of subjective weights. In addition, compared with the MADM methods in the literature, the proposed MADM method can get more accurate decision results.

\section{Conclusions}

In this paper, we extended the HFLTS to the IV2THFLTS. Then we defined some aggregation operators for IV2THFLEs. To overcome the instability of subjective weights, a method was proposed to obtain the weights of attributes based on the differences between attributes. Moreover, we applied the GIV2THFLWA operator to develop a method for MADM problems under the IV2THFL environment. We applied the proposed method to solve an illustrative example where different values of $\lambda$ were used. Finally, the proposed method was compared with other two representative MADM methods. The results showed that the method we presented can avoid information loss and enhance the accuracy of decision results. What is more, it allows DMs to choose different values of $\lambda$ to aggregate linguistic information. Thus, DMs can select the most appropriate parameter according to their preference.

In the future, the proposed MADM method can be utilized in various fields, such as transportation, logistics, and artificial intelligence. Some new weight determination methods may be developed.

\section{Acknowledgments}

The work was supported in part by the National Natural Science Foundation of China (Nos. 71501135, 71771156), the China Postdoctoral Science Foundation (2016T90863, 2016M602698), the Fundamental Research Funds for the central Universities (No. YJ201535), and the Scientific Research Foundation for Excellent Young Scholars at Sichuan University (No. 2016SCU04A23).

\section{References}

[1]V. Torra, Hesitant fuzzy sets. International Journal of Intelligent Systems 25(6) (2010) 529-539.

[2]H.C. Liao and Z.S. Xu, Hesitant Fuzzy Decision Making Methodologies and Applications. Springer, 2017.

[3]H.C. Liao, Z.S. Xu and M.M. Xia, Multiplicative consistency of hesitant fuzzy preference relation and its application in group decision making, International Journal of Information Technology \& Decision Making, 13(1) (2014) 47-76.

[4]H.C. Liao and Z.S. Xu, A VIKOR-based method for hesitant fuzzy multi-criteria decision making, Fuzzy Optimization and Decision Making, 12(4) (2013) 373-392.

[5]H.F. Liu, Z.S. Xu and H.C. Liao, The multiplicative consistency index of hesitant fuzzy preference relation, IEEE Transactions on Fuzzy Systems 24(1) (2016) 82-93.

[6]J.C.R. Alcantud and V. Torra. Decomposition theorems and extension principles for hesitant fuzzy sets. Information Fusion 41 (2018) 48-56

[7]L.A. Zadeh, The concept of a linguistic variable and its application to approximate reasoning-I. Information Sciences 8(3) (1975) 199-249.

[8]R.M. Rodriguez, L. Martinez and F. Herrera, Hesitant fuzzy linguistic term sets for decision making. IEEE Transactions on Fuzzy Systems 20(1) (2012) 109-119.

[9]H.C. Liao, Z.S. Xu and X. J. Zeng, Qualitative decision making with correlation coefficients of hesitant fuzzy linguistic term sets. Knowledge-Based Systems 76(1) (2015) 127-138.

[10]H.C. Liao, Z.S. Xu, E. Herrera-Viedma and F. Herrera, H esitant fuzzy linguistic term set and its application in deci sion making: A state-of-the art survey. International Journ al of Fuzzy Systems, (2017) in press. DOI: 10.1007/s40815 -017-0432-9

[11]C.P. Wei, N. Zhao and X.J. Tang, Operators and comparisons of hesitant fuzzy linguistic term sets. IEEE Transactions on Fuzzy Systems 22(3) (2014) 575-585.

[12]H.C. Liao and Z.S. Xu, Approaches to manage hesitant fuzzy linguistic information based on the cosine distance and similarity measures for HFLTSs and their application in qualitative decision making. Expert Systems with Applications 42(12) (2015) 5328-5336.

[13]H.C. Liao, Z.S. Xu and X.J. Zeng, Distance and similarity measures for hesitant fuzzy linguistic term sets and their application in multi-criteria decision making. Information Sciences 271 (2014) 125-142. 
[14]H.C. Liao, Z.S. Xu and X. J. Zeng, Hesitant fuzzy linguistic VIKOR method and its application in qualitative multiple criteria decision making. IEEE Transactions on Fuzzy Systems 23(5) (2015) 1343-1355.

[15]F.Y. Zhang, L. Luo, H.C. Liao, T. Zhu, Y.K. Shi and W.W. Shen, Inpatient admission assessment in West China Hospital based on hesitant fuzzy linguistic VIKOR method. Journal of Intelligent \& Fuzzy Systems 30(6) (2016) 3143-3154

[16]R.M. Rodríguez, L. Martínez and F. Herrera, A group decision making model dealing with comparative linguistic expressions based on hesitant fuzzy linguistic term sets. Information Sciences 241(12) (2013) 28-42.

[17]H.C. Liao, L. Y. Yang, and Z.S. Xu, Two new approaches based on ELECTRE II to solve the multiple criteria decision making problems with hesitant fuzzy linguistic term sets. Applied Soft Computing 63 (2018) 223-234.

[18]X.L. Wu, H.C. Liao, An approach to quality function deployment based on probabilistic linguistic term sets and ORESTE method for multi-expert multi-criteria decision making. Information Fusion 43 (2018) 13-26.

[19]H.C. Liao, L.S. Jiang, Z.S. Xu, J.P. Xu and F. Herrera, A linear programming method for multiple criteria decision making with probabilistic linguistic information. Information Sciences 415-416 (2017) 341-355.

[20]H. Wang, Extended hesitant fuzzy linguistic term sets and their aggregation in group decision making. International Journal of Computational Intelligence Systems 8(1) (2014) 14-33.

[21]Z.M. Zhang and C. Wu, Hesitant fuzzy linguistic aggregation operators and their applications to multiple attribute group decision making. Journal of Intelligent \& Fuzzy Systems 26(5) (2014) 2185-2202.

[22]Z.S. Chen, K.S. Chin, Y.L. Li and Y. Yang, Proportional hesitant fuzzy linguistic term set for multiple criteria group decision making. Information Sciences 357(C) (2016) 61-87.

[23]R. Lin, X.F. Zhao and G.W. Wei, Models for selecting an ERP system with hesitant fuzzy linguistic information. Journal of Intelligent and Fuzzy Systems 26(5) (2014) 2155-2165.

[24]G.W. Wei, Interval valued hesitant fuzzy uncertain linguistic aggregation operators in multiple attribute decision making. International Journal of Machine Learning \& Cybernetics 7(6) (2016) 1099-1114.

[25]J.Q. Wang, J.T. Wu, J. Wang, H.Y. Zhang and X.H. Chen, Interval-valued hesitant fuzzy linguistic sets and their applications in multi-criteria decision-making problems. Information Sciences 288(C) (2014) 55-72.

[26]F.Y. Meng, C. Wang and X.H. Chen, Linguistic interval hesitant fuzzy sets and their application in decision making. Cognitive Computation 8(1) (2016) 52-68.

[27]F.Y. Meng, X.H. Chen and Q. Zhang, Multi-attribute decision analysis under a linguistic hesitant fuzzy environment. Information Sciences 267(13) (2014) 287-305.

[28] C.X. Zhu, L. Zhu and X.Z. Zhang, Linguistic hesitant fuzzy power aggregation operators and their applications in multiple attribute decision-making. Information Sciences 367-368 (2016) 809-826.

[29]Z.S. Xu and Q.L. Da, The uncertain OWA operator. International Journal of Intelligent Systems 17(6) (2002) 569-575.

[30]N. Chen, Z.S. Xu and M.M. Xia, Interval-valued hesitant preference relations and their applications to group decision making. Knowledge-Based Systems 37(2) (2013) 528-540.

[31]Z.S. Xu, An approach based on the uncertain LOG and induced uncertain LOG operators to group decision making with uncertain multiplicative linguistic preference relations. Decision Support Systems 41 (2006) 488-499.

[32]R.R. Yager, On ordered weighted averaging aggregation operators in multicriteria decision making. Readings in Fuzzy Sets for Intelligent Systems 18(1) (1993) 80-87.

[33]N. Chen, Z.S. Xu and M.M. Xia, Interval-valued hesitant preference relations and their applications to group decision making. Knowledge-Based Systems 37(2) (2013) 528-540.

[34]J.Q. Wang, J.J. Peng, H.Y. Zhang, T. Liu and X.H. Chen, An uncertain linguistic multi-criteria group decision-making method based on a cloud model. Group Decision \& Negotiation 24(1) (2015) 171-192. 